\title{
Characterization of Multiaxial Cold Rolled Al6061
}

\author{
${ }^{1}$ Mahesh Kumar Gupta, ${ }^{2}$ Sahil Aggarwal, ${ }^{3}$ Nikhil Seth, ${ }^{4}$ Anant Attri \\ 1,2, 3, 4 department Of Mechanical Engineering, Srm University Ncr Campus, Ghaziabad, India
}

\begin{abstract}
Multi directional Cold rolling of Al 6061 along the transverse and longitudinal direction results in more uniform distribution of the particulates. This rolling is associated with a considerable amount of damage to the particulates. Room temperature tensile tests and Vickers hardness test carried out on the rolled alloy at different reduction ratios showed, significant increase in strength and hardness. However, the test results show that the alloy could be cold rolled up to 50\% reduction without forming any edge crack. Such behaviors of rolled al alloy are analyzed on the basis of change of microstructure from cores to fine results in increase of tensile strength and hardness by increasing the reduction ratio in the cold rolling process.
\end{abstract}

Keywords-Aluminum Alloys, Cold rolling, Al6061.

Date of Submission: 14 April 2017 Date of Accepted: 30 April 2017

\section{INTRODUCTION}

Some modern engineering applications require materials with high strength and stiffness as well as good elevated temperature properties. Various types of metal alloys are being developed to meet such demands. An alloy is a mixture of either pure or fairly pure chemical elements, which forms an impure substance (admixture) that retains the characteristics of a metal. An alloy is distinct from an impure metal. Alloys are made by mixing two or more elements; at least one of which being a metal, A metal that is normally very soft and malleable, such as aluminum, can be altered by alloying it with another soft metal, like copper. Although both metals are very soft and ductile, the resulting aluminum alloy will be much harder and stronger.Aluminum alloy 6061 is one of the most extensively used of the 6000 series aluminum alloys. Its major content other than al is magnesium and silicon.It is a versatile heat treatable extruded alloy with medium to high strength capabilities. The aluminum alloy are increasingly being used in the transport, aerospace, marine, automobile and mineral processing industries, owing to their improved strength, stiffness and wear resistance properties. The present work has been conducted to enhance the mechanical properties and microstructure evolutions of 6061 aluminum alloy after the cold rolling in longitudinal and transverse direction with different reduction ratios. The microstructure evolutions and mechanical properties of Al 6061 alloy were investigated upon at different reduction ratios.6061 is commonly in construction of aircraft structures, such as wings and fuselages, yacht construction, including small utility boats, Automotive parts, such as the chassis of the Audi A8, tactical flashlights.

\section{DETAILS EXPERIMENTAL}

Aluminum alloy 6061 is a medium strength, heattreatable automotive/aerospace alloy developed by ALCOA in the early 1980s. The chemical composition of 6061 alloy is listed in table1. The primary alloying elements are magnesium, silicon. Addition of manganese controls the grain structure, which results in superior strength.

Physical Properties: Density: 2.7 g/cm3, Melting Point: Approx. 580 ${ }^{\circ} \mathrm{C}$, Modulus of Elasticity: $70-80 \mathrm{GPa}$, Poisson's Ratio: 0.33 .

Table1: Chemical Composition of AL2021

\begin{tabular}{|l|l|l|}
\hline Component & Amount (wt.\%) & \\
\hline Aluminum & Balance & \\
\hline Magnesium & $0.8-1.2$ & \\
\hline Silicon & $0.4-0.8$ & \\
\hline Iron & Max. 0.7 & \\
\hline Copper & $0.15-0.40$ & \\
\hline Zinc & Max. 0.25 & \\
\hline Titanium & Max. 0.15 & \\
\hline Manganese & Max. 0.15 & \\
\hline Chromium & $0.04-0.35$ & \\
\hline Others & 0.05 & \\
\hline
\end{tabular}




\subsection{ROLLING}

The current rolling i.e. the multi-dimensional rolling(owing its name to the alternate longitudinal and transverse direction of rolling is performed under different reduction ratios AL 6061 samples were cut into $5 \mathrm{~cm}$ $x 5 \mathrm{~cm}$ with thickness of $12 \mathrm{~mm}$. These samples were rolled in room temperature $\left(25^{\circ} \mathrm{C}\right)$. The specimens were fed through the rolls, and taken from the outlet channel, all rolling schedules were performed without lubrication and in an asymmetrical configuration, and i.e. the lower table was exactly at the same level as the lower roll, which implies that the friction coefficients are not strictly the same on both rolls. Rolling is done till crack appears, the details are shown in Table2.

Table 2: Rolling Parameter

\begin{tabular}{|l|l|l|l|}
\hline Reduction ratio/thickness & $10 \%$ & $20 \%$ & $40 \%$ \\
\hline Initial (mm) & 11.6 & 11.4 & 11.4 \\
\hline Final (mm) & 10.56 & 9.6 & 6.8 \\
\hline
\end{tabular}

Then the rolled samples were prepared for the different experimental tests like Tensile, Hardness, Optical, and XRD for various results.

\subsection{TENSILE TEST}

One of the very basic tests performed on the mechanical properties of a material, the tensile test is used to determine the amount of tensile stress a material can endure before its failure or breaking under the applied load. The dimension of specimen is prepared is given in figl.

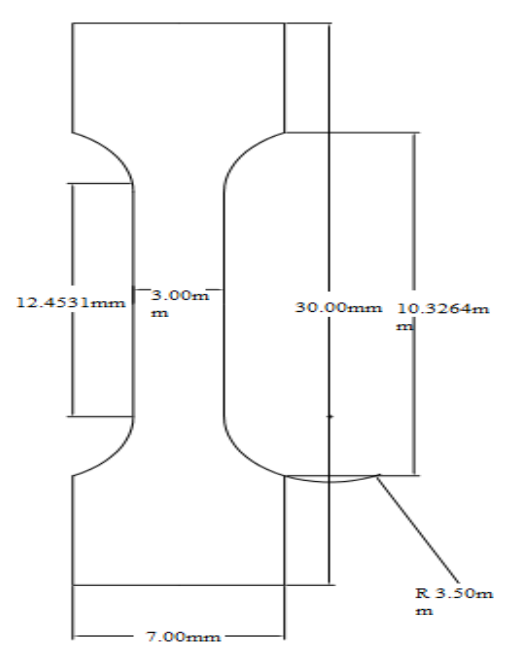

Fig.1.Dimensions of tensile specimen

These test help in determining the changes observed in the subsequent reductions of material.

\subsection{HARDNESS TEST}

Micro hardness was calculated by using Micro-hardness Vickers's testing machine. A precision diamond indenter is impressed on material at a load of $225 \mathrm{mN}$ for 15 secs. Six indentations were done at multiple locations to ensure repeatability of results. In order to avoid the segregation effect of the particles, five readings were taken for each sample and the repeatability value.

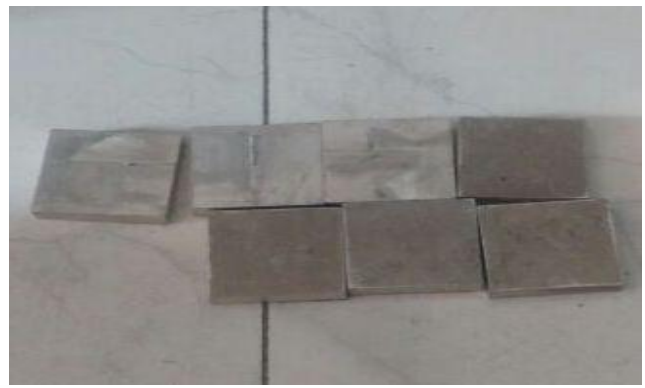

Fig.2. Specimen before rolling 


\subsection{MICROSTRUCTURES EVALUATIONS}

The microstructure was investigated by optical microscopy.For this the samples were polished at different stages ,the samples were cut into small pieces and to remove surface layers damages, grinding is done on it at different grades of silicon carbide paper ranging from grit no 1000,1200, 1500, 2000. Each grinding stage removes the scratches from the previous coarser paper. This is more easily achieved by orienting the specimen perpendicular to the previous scratches, and watching for these previously oriented scratches to be obliterated, then it is finally polished with alumina powder with particle size 1 micron in diameter. The optical microstructures were observed to evaluate the change in grain structures of rolled samples with the standars Al6061 sample.

\section{RESULTS AND DISCUSSION}

\subsection{HARDNESS}

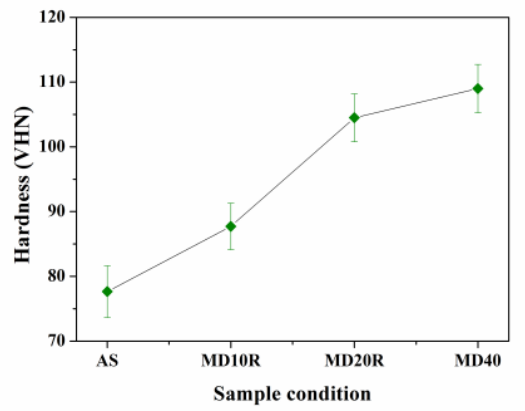

Fig.3. Changes in Vickers hardness at different reduction ratio

Fig. 3 shows the changes in Vickers hardness at different reduction ratios. The alloy tends to increase in hardness after the subsequent reductions under cold rolling operations. Increase in hardness can be seen in Table3.

Table 3: Hardness readings

\begin{tabular}{|l|l|l|l|l|}
\hline S.NO & SAMPLE & MD10R & MD20R & MD40R \\
\hline $\mathbf{1}$ & 69.7 & 89.4 & 103 & 105 \\
\hline $\mathbf{2}$ & 78.3 & 89.4 & 107 & 109 \\
\hline $\mathbf{3}$ & 80.3 & 81.8 & 100 & 107 \\
\hline $\mathbf{4}$ & 79 & 87 & 108 & 109 \\
\hline $\mathbf{5}$ & 78.3 & 91 & 105 & 115 \\
\hline MEAN & $\mathbf{7 7 . 6 5}$ & $\mathbf{8 7 . 7 2}$ & $\mathbf{1 0 4 . 5}$ & $\mathbf{1 0 9}$ \\
\hline
\end{tabular}

\subsection{MICROSTRUCTURE}

The microstructural evolution of 6061 alloy in the process of multi directional cold rolling at different reduction ratios was experimentally studied. Fig4 shows the evolution in the microstructures of the al6061 after different rolling ratios.

It can be seen fromfig4(a)that the grains of 6061 alloy are cores in nature After cold rolling with different reduction ratio, grains were broken up to fine blocks the grains as shown in fig $4(b)$, the alloy grain were elongated and tended to be oriented along the rolling direction since rolling is done on both the directions the grains elongates in both the directions. As the reduction ratio increases the grains broke into more fine blocks it can be seen from fig4(c).

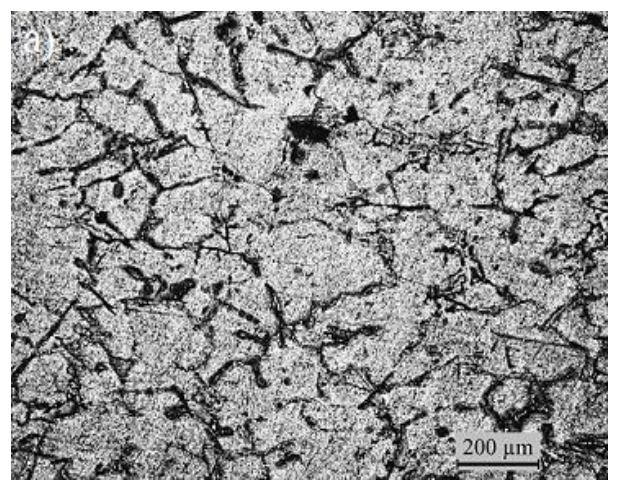



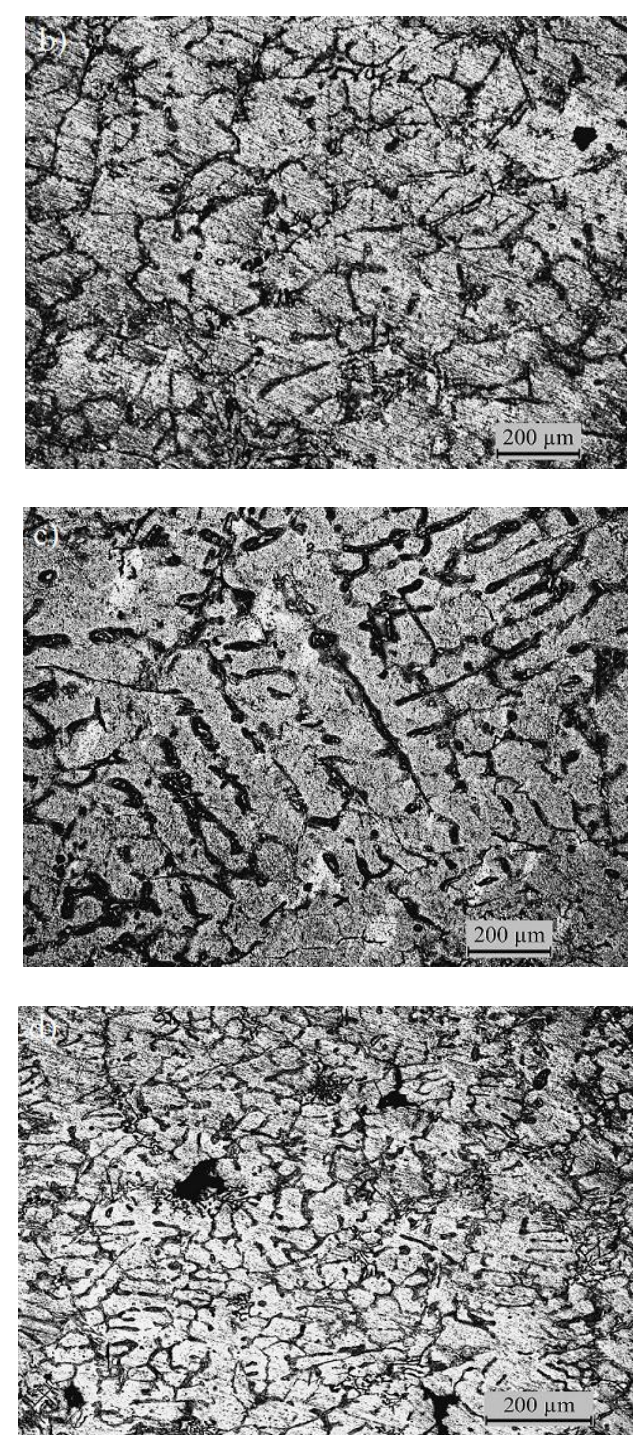

Fig.4. Optical microstructures of AL6061 at different reduction ratio, a) Standard sample b) MD10R c) d) MD40R

MD20R

\subsection{TENSILE PROPERTIES}

The tensile test was performed at room temperature at the different rolled samples. From the data received as shown in Table-4shows that the tensile strength of the material is continuously increasing with increase in reduction ratio.

Table4: Tensile test readings

\begin{tabular}{|c|c|c|c|c|}
\hline Sample & $\begin{array}{l}\text { Maximum } \\
\text { Load [N] }\end{array}$ & $\begin{array}{l}\begin{array}{l}\text { Modulus } \\
\text { modulus) }\end{array} \\
{[\mathrm{MPa}]}\end{array}$ & $\begin{array}{lr}\text { Tensile } & \text { stress } \\
\text { at } & \text { Yield } \\
\text { (Offset } & 0.2 \\
\text { \%) }[\mathrm{MPa}] & \\
\end{array}$ & $\begin{array}{ll}\text { Tensile } & \text { stress at } \\
\text { Break } & \text { (Standard) } \\
{[\text { MPa] }} & \end{array}$ \\
\hline Standard & 2209.87 & 7207.63 & 63.37 & 28.75 \\
\hline MD10R & 2356.47 & 7456.98 & 87.65 & 41.96 \\
\hline MD20R & 2478.32 & 7724.65 & 105.63 & 98.63 \\
\hline MD40R & 2563.56 & 7856.83 & 127.342 & 139.43 \\
\hline
\end{tabular}

\section{CONCLUSIONS}

The presentstudy shows the enhancement of mechanical properties due to microstructural evolution in Al6061 after multidirectional cold rolling at room temperature. The results shows that multi-dimensional rolling generally induces greater increase in strength. 
1. After cold rolling with different reduction ratio,grains were broken up to fine blocks the grains andas the reduction ratio increases the grains broke into more fine blocks and uniformly distributed.

2. The alloy tends to increase in hardnessup to $109 \mathrm{VHN}$ after the subsequent reductions under cold rolling operations.

3. The tensile strength of the material is continuously increasing with increase in reduction ratio and he material can stand up to $2563 \mathrm{~N}$.

\section{ACKNOWLEDGMENTS}

We express our deep sense of indebtedness to Mr. Mahesh Kumar Gupta, our mentor and guide for his excellence guidance and encouragement towards the completion of the project.

We also express our sincere gratitude towards Mr. Freedon Daniel, Head of Department, Mechanical Engineering and SRM University for constant support throughout the project.

\section{REFERENCES}

[1]. Mostafa mansourinejad1, Bahman mirzakhani2 Influence of sequence of cold working and aging treatment on mechanical behavior of 6061 aluminum alloy.

[2]. P. Nageswara Raoa, B. Viswanadhb, R. Jayaganthana Effect of cryorolling and warm rolling on precipitation evolution in Al 6061 alloy(2015).

[3]. Na Wang, Zhimin Zhou and Guimin Lu,Microstructural Evolution of 6061 Alloy during Isothermal Heat Treatment.

[4]. G VenkateshwarluAं*, A.M.K.Prasad $\dot{A}$ and K Ramesh KumarB Evaluation of Mechanical Properties of Aluminium Alloy AA 6061(HE-20).

[5]. Kadirgama K., M.M. Noor, N.M. Zuki N.M., M.M. Rahman, M.R.M. Rejab, R. Daud, K.A. Abou-El-Hossein, (2008), Optimization of Surface Roughness in End Milling on Mould Aluminium Alloys (AA6061T6)Using Response Surface Method and Radian Basis Function Network, Jordan Journal of Mechanical and Industrial Engineering, vol. 2, no. 4, pp. 209-214.

[6]. M. R. Clinch, S. J. Harris, W. Hepples, N. J. H. Holroyd and J. V. Wood: Proc. 6th Int. Conf. on Aluminum Alloys (edited by T. Sato et al., Toyohashi, Japan, 1998) pp. 1185-1190.

[7]. Niranjani v l, hari kumar k c, subramanya sarma v. Development of high strength Al-Mg-Si AA6061 alloy through cold rolling and ageing [J]. Materials Science and Engineering A, 2009, 515: 169-174.

[8]. DEMIR H, GUNDUZ S. The effects aging on machinability of 6061 aluminum alloy [J]. Materials and Design, 2009, 30: $1480-1483$. 